\title{
Caracterização geofísica da região central de Minas Gerais (Belo Horizonte, Curvelo, Três Marias e Bom Despacho).
}

*Jadson da Silva Lima1; Mônica Giannoccaro Von Huelsen'Cláudia Domingues Teixeira.

UUniversidade de Brasília

Copyright 2014, SBGf - Sociedade Brasileira de Geofísica

Este texto foi preparado para a apresentação no VI Simpósio Brasileiro de Geofísica, Porto Alegre, 14 a 16 de outubro de 2014. Seu conteúdo foi revisado pelo Comitê Técnico do VI SimBGf, mas não necessariamente representa a opinião da SBGf ou de seus associados. É proibida a reprodução total ou parcial deste material para propósitos comerciais sem prévia autorização da SBGf.

\section{Abstract}

This work presents the geophysical characterization of the central region of Minas Gerais, with focus on processing and interpretation of Gammaspectrometric data, analysing the false color ternary composition $(R G B)$, where we can note the relationship between the three radioelements $\mathrm{K}$, eU and eTh and delimit regions with similar gammaspectometric signature; and Magnetometric data by analyzing the image of the Amplitude of Analytic Signal, featuring domains and magnetic lineaments, and using Euler's method for estimating the depth of these magnetic sources.

\section{Introdução}

A magnetometria se utiliza das propriedades físicas das rochas em subsuperfície para investigação geológica de uma determinada área. Esse método aerolevantado é eficiente na busca de depósitos minerais que contenham minerais magnéticos. Os minerais radioativos utilizados na produção de combustíveis nucleares podem ser pesquisados utilizando-se o método gamaespectrométrico. Esse método também é favorável ao mapeamento geológico, pois diferentes tipos de rochas podem ser reconhecidos fazendo-se a análise das diferentes assinaturas gamaespectrométricas (An Introduction to Geophysical Exploration, 2002).

O objetivo principal do presente trabalho é o de caracterização geofísica da região central de Minas Gerais através do processamento dos dados de Magnetometria e Gamaespectrometria da Área 10 do Programa de Aerolevantamento Geofísico de Minas Gerais, que é um projeto realizado pela CODEMIG (Companhia de Desenvolvimento Econômico de Minas Gerais) em parceria com a CPRM (Serviço Geológico do Brasil).

\section{Localização da Área}

A área de estudo compreende a região central de Minas Gerais (Fig. 1), onde engloba principalmente as Folhas Três Marias (SE-23-Y-B), Curvelo (SE-23-Z-A), Bom Despacho (SE-23-Y-D) e Belo Horizonte (SE-23Z-C) e uma pequena porção das Folhas Guanhães (SE-23-Z-B) e Ipatinge (SE-23-Z-D) no extremo leste e da Folha Divinópolis (SF-23-X-A) no extremo sul.

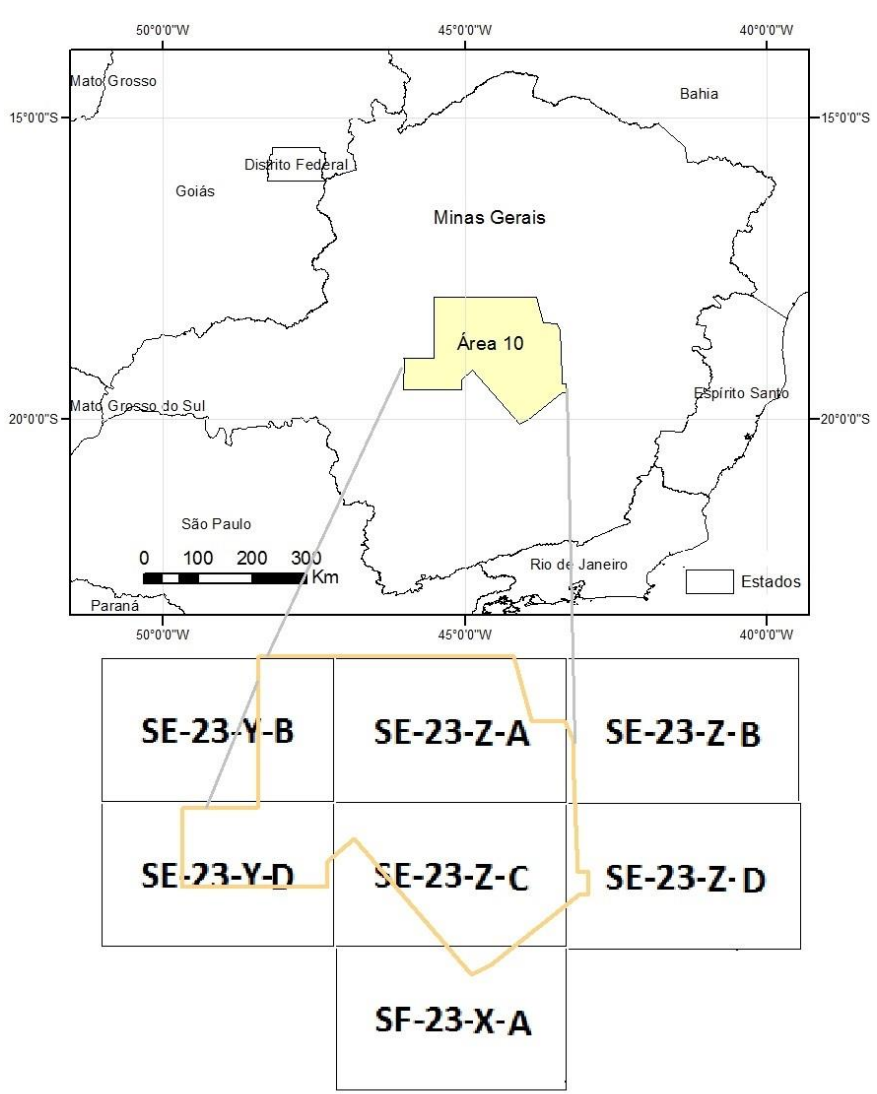

Figura 1 - Mapa da localização da Área de estudo (Área 10 do aerolevantamento), evidenciando sua articulação nas cartas de 1:250.000.

\section{Contexto Geológico}

A maior parte da região está inserida na parte sul do Cráton São Francisco e uma pequena porção se encontra no extremo oeste do Orógeno Araçuaí (Província Mantiqueira), ambos definidos por Almeida (1977). O Cráton é uma unidade geotectônica que ocupa grande parte do território mineiro, apresentandose delimitado pela Faixa Brasília a oeste, e pelo Orógeno Araçuaí a sul e a leste. Almeida (1977) considera que o final do processo de cratonização terse-ia dado durante o Transamazônico. O Orógeno Araçuaí é uma faixa de dobramentos formada devido a colisão do Cráton e Congo.

A geologia da região (Fig. 2; modificado da CPRM, 2003), é caracterizada principalmente por sedimentos das formações Três Marias, Serra da Saudade, Serra de Santa Helena, Sete Lagoas e Lagoa do Jacaré, pertencentes ao Grupo Bambuí que está inserido no Supergrupo São Francisco de idade neoproterozóica. A principal sequência geológica da Província Mantiqueira encontrada na área é a do Espinhaço Meridional, de idade paleo/mesoproterozoica. Dentro dos domínios de bacia 
sedimentar existe 0 predomínio de rochas sedimentares com ocorrência de metamórficas. As rochas sedimentares são representadas, em sua grande maioria, pelos calcários pré-cambrianos do grupo Bambuí. A litologia presente no complexo do Espinhaço apresenta 0 predomínio de rochas metamórficas e ígneas, grande ocorrência de metamorfismo regional, áreas de maciço antigo e escarpamentos orientados por fraturas. Existe 0 predomínio de quartzito, filitos e rochas plutônicas. Os depósitos superficiais, na maior parte inconsolidados, constituem-se de colúvio-eluviais e aluvionares cenozóicos que recobrem extensas áreas.

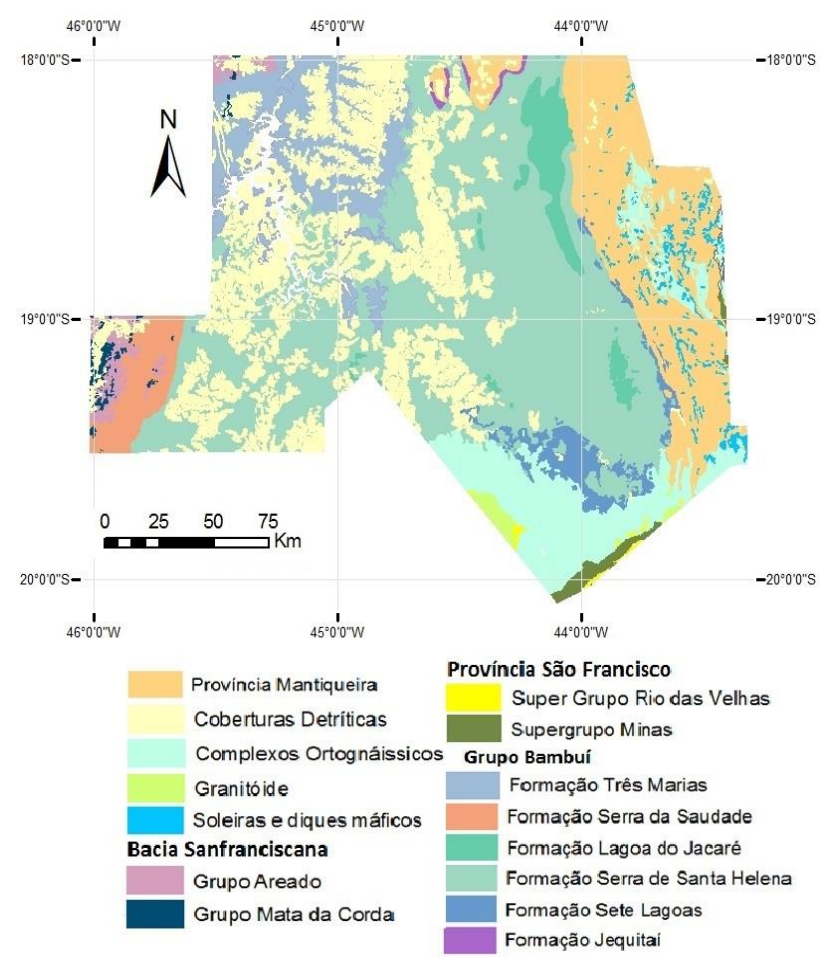

Figura 2 - Mapa Geológico simplificado da área de estudo (modificado da CPRM, 2003).

\section{Metodologia}

No aerolevantamento foram obtidos perfis magnetométricos e gamaespectrométricos de alta resolução com altura média de voo de $100 \mathrm{~m}$ e linhas de voo e controle espaçadas $0,5 \mathrm{~km}$ e $10 \mathrm{~km}$, orientadas nas direções NS e EW, respectivamente.

Para o processamento dos dados e obtenção dos produtos, foi utilizado o programa Oasis Montaj da Geosoft, versão 8.0. Todos os grids foram obtidos através do método interpolador de curvatura mínima com o tamanho das células de $125 \mathrm{~m} \mathrm{(}{ }^{1 / 4}$ da distância entre as linhas de voo).

No processamento dos dados gamaespectrométricos (Fig. 3), primeiramente foi corrigida as concentrações de $\mathrm{K}(\%)$, eU (ppm) e eTh (ppm) baseando-se em suas concentrações médias na crosta terrestre. Em seguida obtivemos os mapas dos respectivos canais corrigidos e do canal de Contagem Total. Também foi adquirida a imagem ternária RGB para análise da relação entre os três radioelementos e delimitação dos domínios gamaespectrométricos.

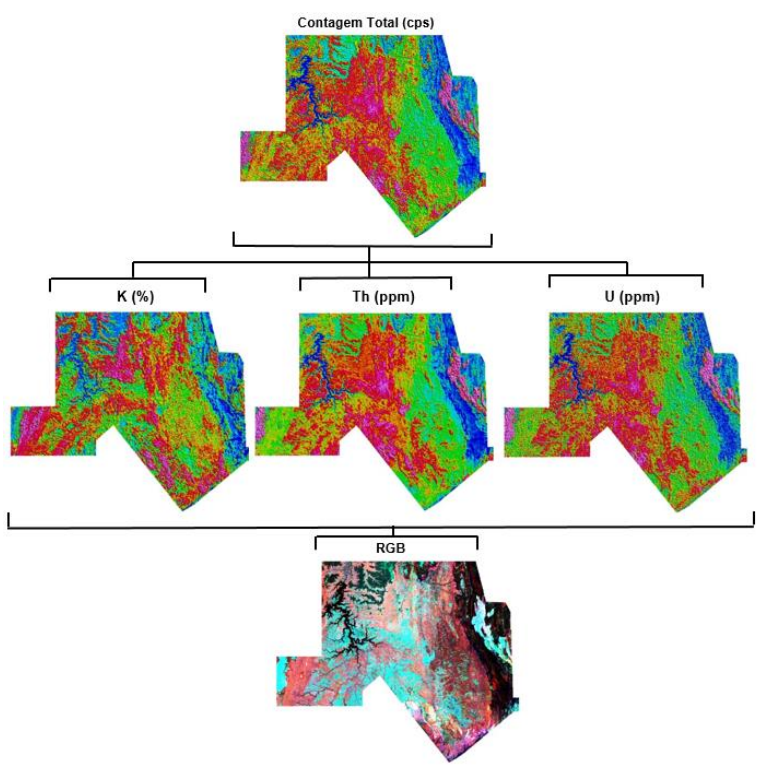

Figura 3 - Fluxograma do processamento dos dados Gamaespectrométricos mostrando os principais produtos obtidos.

As etapas do processamento dos dados magnetométricos da área de estudo são mostrados na Fig. 4. Inicialmente obtivemos o Campo Magnético Anômalo (CMA) retirando-se o IGRF dos dados. A partir do CMA, conseguimos as malhas das derivadas horizontais (DX e DY) e vertical (DZ) para uma observação mais superficial da área e a malha da Amplitude do Sinal Analítico (ASA), para localização horizontal dos corpos magnéticos.

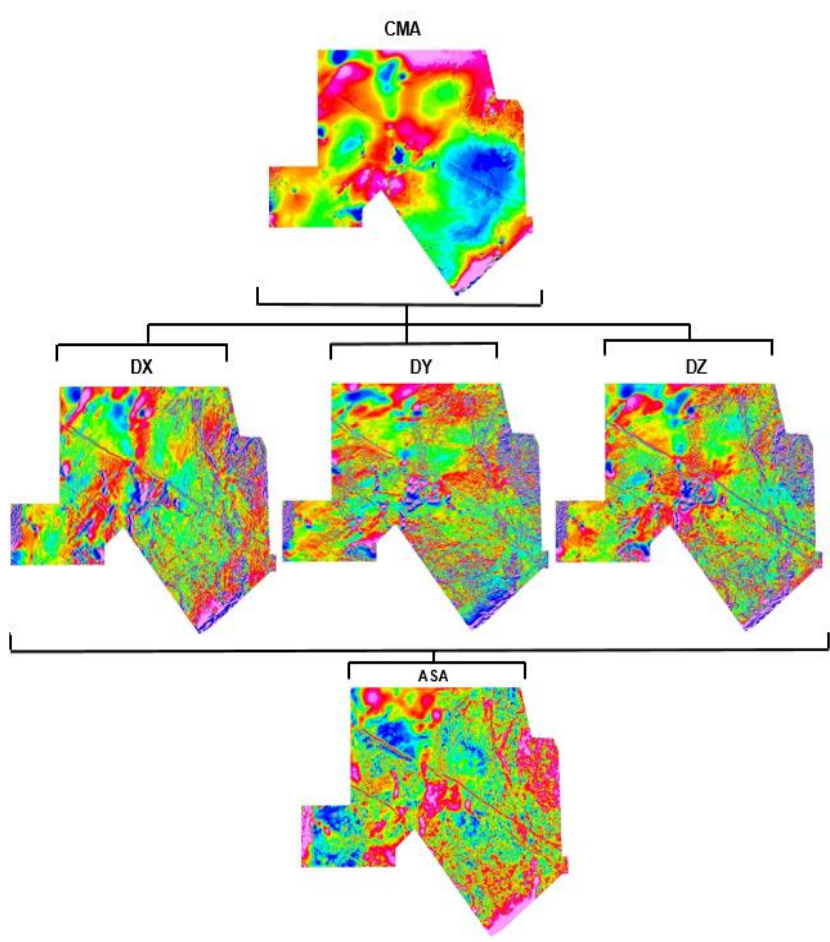

Figura 4 - Fluxograma do processamento magnetométrico com os principais produtos obtidos. 


\section{Resultados}

\section{Interpretação Gamaespectrométrica}

Analisando a imagem do RGB, foram delimitadas regiões com assinaturas gamaespectrométricas semelhantes, contabilizando-se 11 domínios no total (Fig. 5). Observando cada domínio, é possível ter uma estimativa da concentração dos radioelementos $\mathrm{K}(\%)$, eTh (ppm) e eU (ppm) que varia de baixa à alta.

O domínio A (verde escuro) apresenta concentrações médias de eTh e baixas de eU e $\mathrm{K}$, e representa depósitos de areia, argila e coberturas lateríticas. O domínio B (preto) engloba o Lago Três Marias e apresenta praticamente nenhuma resposta gamaespectométrica, pois a água tem a característica de atenuar a energia eletromagnética gama, dificultando a detecção dos radioelementos. O domínio $\mathrm{C}$ (rosa claro) apresenta teores médios de $\mathrm{K}$ e baixos de eTh e eU. A região faz parte do Subgrupo Paraopeba Indiviso do Grupo Bambuí que é caracterizado pela presença de folhelhos e siltitos. O domínio D (azul claro) mostra valores médios de U e Th e baixo de k. A geologia é representada por depósitos de areia e argila, coberturas lateríticas e aglomerados. O domínio E (vermelho claro/escuro) possui elevados teores de potássio e baixos teores de $U$ e Th. Nessa área encontramos a formação Lagoa do Jacaré na maior parte da porção norte com a presença de calcário e siltito e no sul temos a presença de siltito e folhelho. O domínio $F$ (vermelho escuro/preto) apresenta baixa concentração dos três radioelementos. A litologia é característica do Espinhaço Meridional. Os domínios G, $\mathrm{H}$ e I apesar de apresentarem concentrações variadas dos três radioelementos, possuem o mesmo arcabouço geológico que é representada pelos gnaisses. O domínio J (vermelho) é caracterizado pelo alto teor de $\mathrm{K}$ e baixo de eU e eTh. Essa região faz parte da Formação da Serra da Saudade do Subgrupo Paraopeba indiviso (Grupo Bambuí), cuja litologia característica são os siltitos, argilitos e arenito. O domínio $\mathrm{K}$ (azul) possui altos teores eTh e eU e baixo de K. É onde se localiza o Grupo Mata da Corda, que é caracterizado pela presença de lavas e rochas piroclásticas.

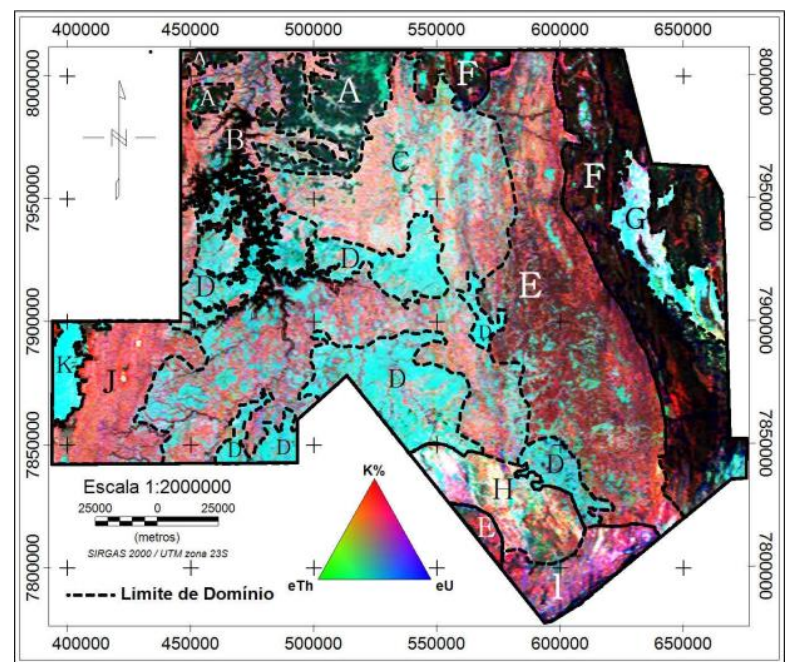

Figura 5 - $\quad$ Mapa da interpretação Gamaespectométrica obtida através da composição ternária de falsa cor RGB, contabilizando 11 domínios.

\section{Interpretação Magnetométrica}

A interpretação magnetométrica da área de estudo baseou-se principalmente na delimitação dos domínios e lineamentos magnéticos, e a partir desses resultados, caracterizamos a área em 4 grandes regiões (Fig 6). Os domínios magnéticos foram obtidos de acordo com a imagem do produto do sinal analítico (ASA) em escala logarítmica, obtendo-se assim 3 domínios. O domínio rosa possui valores que variam de 0,03 à 0,09 $\mathrm{nT} / \mathrm{m}$, o amarelo está entre 0,005 á $0,021 \mathrm{nT} / \mathrm{m}$ e o domínio verde apresenta valores bem próximos a zero. Os lineamentos magnéticos foram delimitados tendo-se como base a imagem do ASA, e com a ajuda das imagens da Inclinação do Sinal Analítico (ISA) e da derivada vertical $(\mathrm{Dz})$.

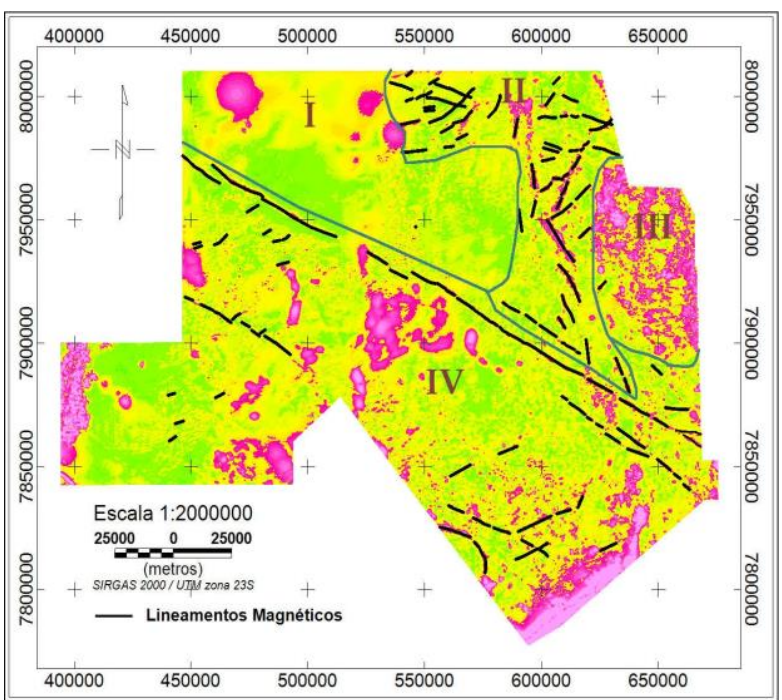

Figura 6 - Mapa dos domínios e lineamentos magnéticos interpretados principalmente da imagem da Amplitude do Sinal Analítico.

As estimativas das profundidades das estruturas e corpos magnéticos foram realizadas utilizando a deconvolução de Euler (Fig. 8 e 9), que é uma técnica desenvolvida por Thompson (1982), estendida para uma situação tridimensional por Reid et al (1990) e se traduz na seguinte equação:

$$
\left(x-x_{0}\right) \frac{\partial}{\partial x} T+\left(y-y_{0}\right) \frac{\partial}{\partial y} T+\left(z-z_{0}\right) \frac{\partial}{\partial z} T=-\eta T
$$

Onde temos que uma fonte tridimensional situada na posição ( $\mathrm{x} 0, \mathrm{y} 0, \mathrm{z} 0)$, produz uma anomalia de campo magnético total $(T)$. O valor $\eta$ varia de 0 a 3 e é chamado de índice estrutural, que por sua vez é escolhido em função do que queremos observar em subsuperfície (contatos, diques, corpos, etc.). 


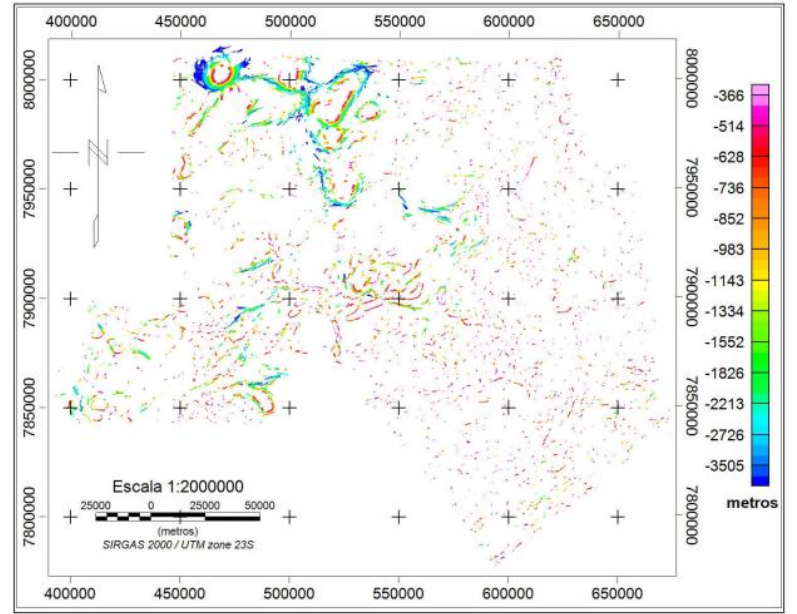

Figura 8 - Mapa com os resultados da deconvolução de Euler para o valor de índice estrutural 0.

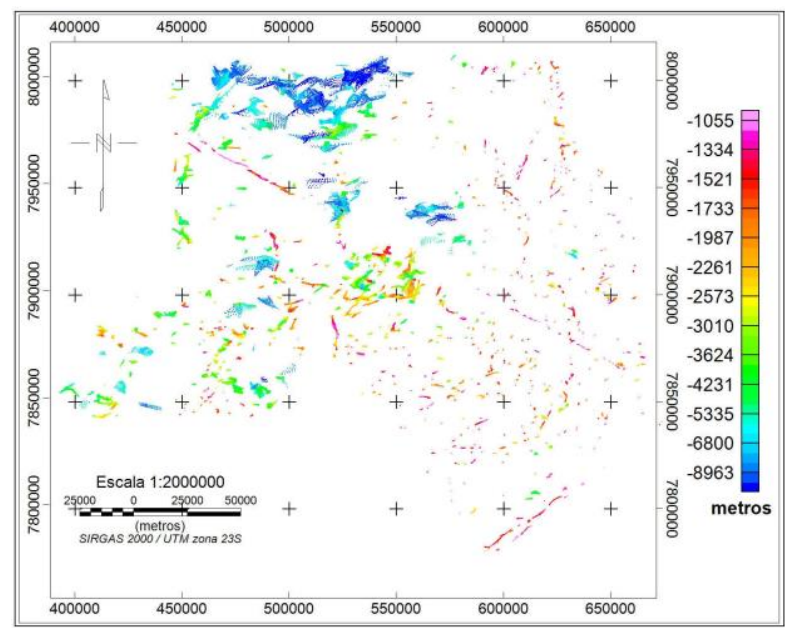

Figura 9 - Mapa com os resultados da deconvolução de Euler para o valor de índice estrutural 2.

As estruturas magnéticas demarcadas (Fig. 6) estão orientadas preferencialmente nas direções SW-NE e SE-NW, sendo que o maior número de ocorrência desses lineamentos se localiza no setor N75W, como podemos ver no histograma polar da Fig. 10.

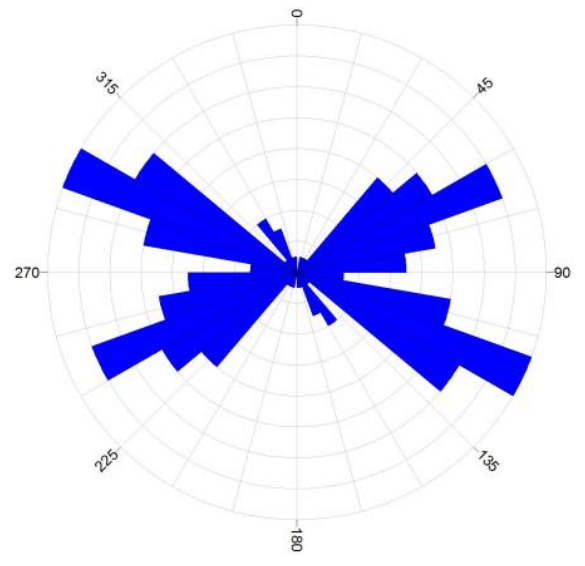

Figura 10 - Diagrama de rosetas obtido a partir dos lineamentos magnéticos.
Como podemos observar na Fig. 6, a região I é caracterizada principalmente por valores baixos de campo magnético, mas apresenta um corpo anômalo à noroeste, de formato arredondado com diâmetro médio de $1200 \mathrm{~m}$ e profundidade que varia de centenas de metros em seu centro a milhares de metros em sua borda (Fig. 8). Não há evidências geológicas à cerca desse corpo. A característica marcante observada na região II é a densidade de estruturas magnéticas orientadas principalmente nas direções SW-NE e SENW. A região III é caracterizada por um relevo magnético movimentado que dificulta a observação de estruturas magnéticas apesar de na mesma região observarmos a presença de soleiras e diques máficos. A região IV apresenta grandes anomalias magnéticas na região central, oeste e sudeste e poucas estruturas magnéticas orientadas nas direções SW-NE e SE-NW, mas a de maior expressão é a que corta toda a região no sentido SE-NW com profundidades que variam de $1000 \mathrm{~m}$ à $2000 \mathrm{~m}$ (Fig. 9).

\section{Discussão e Conclusões}

Neste trabalho observamos a grande correlação dos domínios gamaespectrométricos com o mapa geológico dá área. Essa correlação se dá principalmente no domínio $A$, que representa coberturas detríticas; $B$, onde encontramos o Lago Três Marias, ou seja, sem resposta gamaespectrométrica; e o F que seria o Espinhaço Meridional. A magnetometria mostra que os corpos com maiores amplitudes magnéticas da parte norte e central do mapa apresentam maiores profundidades e a estrutura magnética que corta a região central com direção NW$\mathrm{SE}$, é rasa. Para trabalhos futuros será obtido a continuação para cima, sobreposição ao mapa gamaespectrômetrico o modelo digital de terreno, além de uma análise mais detalhada da área.

\section{Agradecimentos}

Agradecemos à Companhia de Desenvolvimento Econômico de Minas Gerais (CODEMIG) pela liberação dos dados aerogeofísicos utilizados no presente trabalho. O primeiro autor agradece ao programa PIBIC/CNPQ pela concessão da bolsa de iniciação científica (2013/2014).

\section{Referências}

ALMEIDA, F. F. M. 1977. O Cráton do São Francisco. Revista Brasileira de Geociências. 7: 349-364.

CPRM - Serviço Geológico do Brasil. Mapa Geológico do Estado de Minas Gerais, 2003. Disponível em:http://www.cprm.gov.br/

KEAREY, P., BROOKS, M. \& HILL, I. (2002) An introduction to Geophysical Exploration. Blackwell Scientific. Oxford (UK). 262p.

REID, A. B; ALLSOP, J. M; GRANSER, H; MILLETT, A. J; SOMERTON, I. W. 1990. Magnetic interpretation in three dimensions using Euler deconvolution. Geophysics. 55:80-91.

THOMPSON, D. T. 1982. EULDPH: A new technique for making computer assisted depth estimates from magnetic data. Geophysics, 47: 31-37. 